\title{
Mouse Mammary Tumor Virus Promoter-Containing Retroviral Promoter Conversion Vectors for Gene-Directed Enzyme Prodrug Therapy are Functional in Vitro and in Vivo
}

\author{
Reinhard Klein, ${ }^{1,2}$ Bärbel Ruttkowski, ${ }^{3,4}$ Sonja Schwab, ${ }^{3}$ Thomas Peterbauer, ${ }^{5}$ Brian Salmons, ${ }^{1}$ \\ Walter H. Günzburg, ${ }^{3}$ and Christine Hohenadl ${ }^{1,3}$ \\ ${ }^{1}$ Austrianova Biotechnology GmbH, Veterinärplatz 1, A-1210 Vienna, Austria \\ ${ }^{2}$ Children's Cancer Research Institute, Kinderspitalgasse 6, A-1090 Vienna, Austria \\ ${ }^{3}$ Research Institute of Virology and Biomedicine, University of Veterinary Medicine, Veterinärplatz 1, A-1210 Vienna, Austria \\ ${ }^{4}$ Department of Pathobiology, University of Veterinary Medicine, Veterinärplatz 1, A-1210 Vienna, Austria \\ ${ }^{5}$ Department of Pharmacology and Toxicology, University of Vienna, Althanstraße 14, A-1090 Vienna, Austria
}

Correspondence should be addressed to Reinhard Klein, reinhard.klein@ccri.at

Received 1 October 2007; Accepted 14 December 2007

Recommended by Kanury V. S. Rao

Gene directed-enzyme prodrug therapy (GDEPT) is an approach for sensitization of tumor cells to an enzymatically activated, otherwise nontoxic, prodrug. Cytochrome P450 2B1 (CYP2B1) metabolizes the prodrugs cyclophosphamide (CPA) and ifosfamide (IFA) to produce the cytotoxic substances phosphoramide mustard and isophosphoramide mustard as well as the byproduct acrolein. We have constructed a retroviral promoter conversion (ProCon) vector for breast cancer GDEPT. The vector allows expression of CYP2B1 from the mouse mammary tumor virus (MMTV) promoter known to be active in the mammary glands of transgenic animals. It is anticipated to be used for the generation of encapsulated viral vector producing cells which, when placed inside or close to a tumor, will act as suppliers of the therapeutic CYP2B1 protein as well as of the therapeutic vector itself. The generated vector was effectively packaged by virus producing cells and allowed the production of high levels of enzymatically active CYP2B1 in infected cells which sensitized them to killing upon treatment with both IFA and CPA. Determination of the respective $\mathrm{IC}_{50}$ values demonstrated that the effective IFA dose was reduced by sixteen folds. Infection efficiencies in vivo were determined using a reporter gene-bearing vector in a mammary cancer cell-derived xenograft tumor mouse model.

Copyright (c) 2008 Reinhard Klein et al. This is an open access article distributed under the Creative Commons Attribution License, which permits unrestricted use, distribution, and reproduction in any medium, provided the original work is properly cited.

\section{INTRODUCTION}

Conventional cancer chemotherapy including chemotherapy of breast cancer often results in severe systemic toxicity at drug concentrations necessary for effective killing of tumor cells. This obstacle can be overcome with the concept of gene-directed enzyme prodrug therapy (GDEPT) that implies selective delivery into tumor cells and expression of drug-metabolizing transgenes within them [1].

The oxazaphosphorine cyclophosphamide (CPA) and its structural isomer ifosfamide (IFA) are DNA-alkylating agents commonly used in breast cancer chemotherapy [2]. These anticancer agents are administered as prodrugs that are primarily activated by the hepatic enzyme cytochrome P450 (CYP). Among the P450 enzymes, the subfamily 2B en- zymes CYP2B1 (from rat) and CYP2B6 (from human) have been shown to be the most active catalysts for this enzymatic reaction [3, 4]. The generated anticancer metabolites phosphoramide mustard (from CPA) or isophosphoramide mustard (from IFA) as well as acrolein are systematically distributed throughout the body eventually reaching the tumor but also causing undesired toxic side effects. Local activation of cyclophosphamide or ifosfamide at the site of the tumor would allow to use lower concentrations of the prodrug resulting in lower systemic toxicity with a still effective or, if using conventional prodrug dosages, a much more potent cell killing effect on the tumor cells. In addition, cyclophosphamide and ifosfamide suicide gene therapy has the advantage of also exerting a bystander effect as it causes the death of 
not only the therapeutic transgene-carrying cells but also of neighboring nontransgenic cells via passive diffusion of the cytotoxic metabolites $[5,6]$.

Gene therapy requires strong and, if possible, selective expression of the transgene in the requested tissue or organ. For mammary gland-specific expression of transgenes in mammals, a number of promoters from various sources have been evaluated. Among those are the promoters of milk proteinencoding genes such as the whey acidic protein (WAP), $\beta$ lactoglobulin, $\alpha$-s1-casein, $\beta$-casein, or the C3(1) promoter [7]. However, for mouse models of human breast cancer, the long terminal repeat (LTR) of the mouse mammary tumor virus (MMTV) has emerged as the most potent and frequently used promoter to drive transgene expression [8-12]. It, therefore, also represents one of the major candidate promoters for human breast cancer gene therapy. The MMTV promoter is most active during lactation due to induction by lactogenic hormones such as prolactin $[13,14]$. However, the most potent inducing effects are due to the presence of hormone response elements (HREs) within the $\mathrm{U} 3$ region of the viral LTR that respond to androgens, progestins, mineralocorticoids, and glucocorticoids [15].

For in vitro and in vivo transgene delivery, a number of techniques have been elaborated. Among those, infection with retroviral vectors represents a very efficient method. Due to their capacity to integrate into the host genome, retroviral vectors are one choice if long-term gene expression of a transgene is desired and thus they have been used in a variety of gene therapy studies [16]. However, to date, several rounds of vector delivery are necessary to achieve satisfactory transfer of a therapeutic gene in vivo. This is mainly due to unsatisfactory virus titers, rapid clearance of the vector by the liver and the spleen [17], and, in the case of MLV-based retroviral vectors, the fact that only dividing cells can be reached, thereby a priori limiting the number of accessible cells [18]. In addition, repeated delivery may cause the risk of the development of an immune response against the vector, thereby impairing gene transfer. The establishment of an in situ cell depot, constantly producing therapeutic retroviral vectors where required, may overcome those hurdles and may allow much more efficient delivery of the transgene. In previous experiments, for proof of principle, we have encapsulated virus packaging cells that not only expressed a reporter gene from a retroviral vector but also produced virus particles. Upon insertion into the mammary glands of mice, the virus particles were liberated from the capsules and transferred the reporter gene to surrounding cells [19]. This type of delivery has also been shown by others to be functional using a potentially therapeutic suicide gene for the treatment of glioblastoma [20], and similar long-term in vivo gene delivery could also be achieved using TheraCyte immuno-isolation devices containing spleen necrosis retrovirus packaging cells [21]. We have also shown previously that, when combined with ifosfamide-based chemotherapy, intratumoral injection as well as instillation of capsules containing CYP2B1-expressing (but not virus-producing) cells into tumor-supplying blood vessels leads to a significantly increased tumoricidal effect on experimentally generated tumors in mice $[22,23]$ or on inoperable pancreatic tumors in humans $[24,25]$.

In this study, we have constructed MLV-based replication-deficient retroviral promoter conversion (ProCon) vectors for the use in breast cancer gene therapy in combination with the in situ vector supply and delivery system described above. The vectors are designed to express the CYP2B1 gene within the packaging cells to convert the prodrugs ifosfamide or cyclophosphamide into their active forms. In addition, the packaging cells produce virus particles that are liberated from the cells and, by infection, can transfer the therapeutic gene into the surrounding target cells. In the vectors described in this study, the original U3 region of the $3^{\prime}$ long terminal repeat (LTR) is replaced with the heterologous MMTV promoter. In virus-packaging cell lines, transcription of the eGFP-encoding reporter gene or of the actual therapeutic CYP2B1-encoding gene is driven by the MLV promoter/enhancer. After infection, in the course of reverse transcription, the heterologous MMTV promoter is duplicated and one copy is translocated to the U3-region of the 5'LTR. This rearrangement finally brings the expression of the transduced gene under control of the MMTV promoter. We show that the genes incorporated into the vectors are efficiently expressed in virus packaging cells as well as in infected cells. Virus titers obtained with the generated vectors are sufficiently high to allow efficient infection of target cells both in vitro and in a murine tumor model in vivo. We further show that the generated therapeutic CYP2B1 protein is enzymatically active and exerts a strong cell killing effect on infected breast cancer cells upon treatment with ifosfamide or cyclophosphamide in vitro.

\section{MATERIALS AND METHODS}

\subsection{Plasmids}

Plasmids pPCCMm1 and pPCEMm1 (see Figure 1) are identical in construction except for the fact that pPCCMm1 carries the Cyp2B1 gene and pPCEMm1 carries the eGFP gene. pPCCMm1 was constructed by replacing a cytomegalovirus (CMV) promoter-harboring MluI-SacII fragment of plasmid pPCCmCMV.WPRE (Harry Holzmüller, Austrianova Biotechnology $\mathrm{GmbH}$ ) with an MluI-SacII fragment from plasmid pPCEMa [26] harboring the MMTV promoter. Plasmid pPCEMm1 was constructed as described elsewhere [27]. pCMV-dsRed-Express was purchased from BD Clontech.

\subsection{Nucleic acid extraction}

Plasmid DNA was isolated using a QIAprep spin miniprep kit (Qiagen, Calif, USA) or a Qiagen-tip 100 plasmid midi kit (Qiagen). Linear DNA fragments were purified using a QIAquick gel extraction kit (Qiagen).

\subsection{Cell lines}

Human 2GP19Talf amphotropic retroviral packaging cells [28], human 293 embryonic kidney cells (ATCC CRL-1573) 


$$
\begin{aligned}
& \text { pPCCMm1 } \\
& \cline { 2 - 3 } \\
& \cline { 2 - 3 }
\end{aligned}
$$

Figure 1: MLV-based retroviral ProCon vectors. 5'LTRs consist of unique 3'(U3), repeated (R), and unique 5'(U5) regions. 3'LTRs consist of repeated (R) and unique $5^{\prime}(\mathrm{U} 5)$ regions. The $3^{\prime}$ LTR U3 region has been replaced by the MMTV promoter that drives expression of the transgene (eGFP or CYP2B1) in infected cells. All vectors contain the CMV enhancer region (CMV enh) upstream of the 5'LTR, a packaging region $(\psi)$, an internal SV40 promoter (SV40) driving the expression of a neomycin resistance gene (neo), the Woodchuck hepatitis virus posttranscriptional regulatory element (WPRE), and an origin of replication (ori) for replication in E. coli.

[29], and feline CRFK kidney cells (ATCC CCL-94) [30] were grown in Dulbecco'smodified Eagle's medium (DMEM)/ Glutamax (Invitrogen Life Technologies, Calif, USA) supplemented with $10 \%$ fetal bovine serum (FBS; Invitrogen Life Technologies). Human T-47D (mammary gland; ductal carcinoma) cells (ATCC HTB-133) [31] were cultivated in DMEM/Glutamax supplemented with 10\% FBS and $6.5 \mu \mathrm{g} / \mathrm{mL}$ insulin (Sigma-Aldrich, Miss, USA).

\subsection{Transfection}

Transfections of 2GP19Talf cells were performed by calcium phosphate coprecipitation according to the instructions of the manufacturer (Amersham Biosciences, NJ, USA). For stable transfections, the transfected cells were selected in medium containing $0.4 \mathrm{mg} / \mathrm{mL}$ Geneticin(G418; Invitrogen Life Technologies) until mock-transfected cells had died. Stably transfected cells were maintained as populations in the presence of $0.4 \mathrm{mg} / \mathrm{mL} \mathrm{G} 418$.

\subsection{In vitro infection experiments}

Culture supernatants from $2 \times 10^{6}$ virus-producing cells were usedto infect $4 \times 10^{5}$ target cells as described elsewhere [28]. For titer calculation, dilutions of infected CRFK cells were trypsinized and replated 24 hours afterinfection in triplicates and selected in medium containing $0.4 \mathrm{mg}$ of G418/mL.After 10 to 14 days of culture, drug-resistant colonies werecounted, and the number of colony-forming units (CFU) per milliliter of vector supernatant wascalculated. Populations of stably infected T-47D and CRFK cells were maintained in the presence of $0.4 \mathrm{mg} / \mathrm{mL} \mathrm{G} 418$. For stimulation of expression, cells were treated with $1 \mu \mathrm{M}$ dexamethasone (Sigma-Aldrich) every 48 hours.

\subsection{In vivo infection experiments}

For establishment of mixed tumors 2GP19Talf/pPCEMm1 cells and T-47D/DsRed or CRFK/DsRed cells were trypsinized out of the culture flasks, washed three times with PBS, and mixed in a ratio of $1: 5$ or $1: 10$. A total number of $6 \times 10^{5}$ mixed cells in a total volume of $100 \mu \mathrm{L}$ RPMI medium containing $100 \mathrm{U} / \mathrm{mL}$ penicillin/streptomycin (Invitrogen Life Technologies) and 50\% matrigel (Becton Dickinson Biosciences, NJ, USA) was injected into the mammary fat pads of Hsd: Athymic Nude-nu mice (Harlan
Winkelmann, Borchen, Germany). For stimulation of tumor growth, slow-release estrogen pellets $(1.13 \mathrm{mg} /$ pellet, 60 days release; Innovative Research of America, Fla, USA) were implanted. At an average tumor size of $100-200 \mathrm{~mm}^{3}$, mice were treated with $500 \mu \mathrm{g}$ dexamethasone/mouse (Voren, $1 \mathrm{mg} / \mathrm{mL}$, Boehringer Ingelheim, Ingelheim, Germany) intraperitoneally on 3 consecutive days. On day 4, tumors were explanted, digested to single-cell suspension by incubation with $2 \mathrm{mg} / \mathrm{mL}$ collagenase (Sigma-Aldrich) in PBS at $37^{\circ} \mathrm{C}$ for two hours, washed twice with DMEM/10\% FBS, and finally analyzed by fluorescence-activatedcell sorting (FACS) and confocal laser-scanning microscopy. In vivo experiments were carried out according to Austrian law regulating animal experimentation (GZ 68.205/109-BrGT/2003).

\subsection{Detection of fluorescent cells}

For detection of DsRed- and EGFP-expressing tissue culture cells, those were trypsinized, washed twice with $\mathrm{PBS} / 10 \%$ FBS, and then 50,000 cells per sample were analyzed for fluorescencewith an FACS analyzer (FACScalibur; Becton Dickinson). Cells from explanted tumors were treated as described above. The numbers and meanfluorescence intensities (MFIs) of positive cells were determined using the CellQuest software (Becton Dickinson). For confocal laserscanning microscopy, a Zeiss LSM 510 inverted microscope equipped with a $40 \mathrm{x}, 1.3$ numerical aperture, oil immersion objective (Plan Neofluar, Zeiss, Göttingen, Germany) was used. Cells analyzed by laser-scanning microscopy were prepared in the same way as for FACS analysis.

\subsection{FACS sorting}

Populations of cells expressing the fluorescent proteins EGFP or DsRed were FACS sorted to exclude nonfluorescent cells before their use in in vivo experiments. Therefore, cells to be sorted were expanded to approximately $3 \times 10^{7}$, harvested by trypsinization, resuspended in normal cell culture medium, and pelleted by cetrifugation at $410 \times \mathrm{g}$ for 5 minutes. Thereafter, cells were washed twice in PBS and resuspended in PBS containing 5\% FBS. Then, the cell suspension was filtered through a sterile nylon gauze into an FACS tube and stored on ice until sorting. FACS sorting was performed using the FACSVantage SE device (Becton Dickinson) which is controlled by the Cell Quest Pro software (Becton Dickinson). 


\subsection{Detection of protein and enzymatic activity}

CYP2B1 was detected by Western blotting using an antiCYP2B1 antibody (Daiichi Pure Chemicals Co., LTD, NJ, USA). The enzymatic activity of CYP2B1 was determined in a 96-well format resorufin assay. Briefly, cells were washed twice with PBS, trypsinized out of the culture flasks, and resuspended in DMEM/Glutamax medium lacking phenol red (Invitrogen Life Technologies) supplemented with 10\% FBS. $100 \mu \mathrm{L}$ of cell suspension containing a total of $4 \times 10^{5}$ cells was pipetted into a black clear bottom 96-well plate in quadruplicates. DMEM/Glutamax medium containing 10\% FBS but lacking phenol red was used as a blank. $5 \mu \mathrm{L}$ of substrate ( $0.3 \mathrm{mM}$ benzylresorufin in DMSO; Sigma-Aldrich) was added to each well. Plates were incubated for 1 hour at $37^{\circ} \mathrm{C} / 5 \% \mathrm{CO}_{2}$ protected from light. Samples were fluorometrically analyzed for the presence of generated resorufin in a plate reader (Tecan Systems) using an extinction wavelength of $520 \mathrm{~nm}$ and an emission wavelength of $590 \mathrm{~nm}$. The CYP2B1 enzymatic activities of a selected single cell clone (22P1G) [32] served as a reference.

\subsection{Cytotoxicity assays}

$1 \times 10^{4} \mathrm{~T}-47 \mathrm{D}$ or $\mathrm{T}-47 \mathrm{D} / \mathrm{pPCCMm} 1$ cells were seeded into 96 well plates in quadruplicates and cultivated in DMEM/ Glutamax medium lacking phenol red (Invitrogen Life Technologies) supplemented with 10\% FBS for six days without changing the medium. 24 hours after seeding of the cells, the medium was supplemented with $0,0.03,0.1,0.3,1$, or $3 \mathrm{mM}$ ifosfamide (Baxter, Ill, USA) or cyclophosphamide (Baxter). The supplementation with dexamethasone was repeated every 48 hours. Five days after incubation with ifosfamide or cyclophosphamide, the viabilities of the cells were determined with an XTT assay according to the instructions of the manufacturer (Promega, Wiss, USA). The plates were analyzed with a plate reader (Tecan Systems Inc., Calif, USA) by reading the absorbance at $490 \mathrm{~nm}$.

\section{RESULTS}

\subsection{The generated ProCon vector system allows efficient infection of target cells}

We have previously evaluated MLV-based ProCon vectors (pPCEM and pPCEMm1) containing the mammary glandspecific MMTV promoter and the eGFP gene as a reporter for their gene expression and infection capacities in vitro [27]. Here, for in vivo investigations of the efficacy of the therapeutic vectors, we chose the following experimental setup: we wanted to determine if efficient infection of target cells is (i) also achievable by cocultivation of virus-producing cells and target cells in vitro instead of using high-titer virus suspensions for in vitro infections and (ii) in a mixed tumor model in vivo, thus mimicking a future therapeutic situation in which viruses would be released from capsules. In 293based virus-producing 2GP19Talf cells, vector pPCEMm1 (see Figure 1) [27] that was used in this study allows the

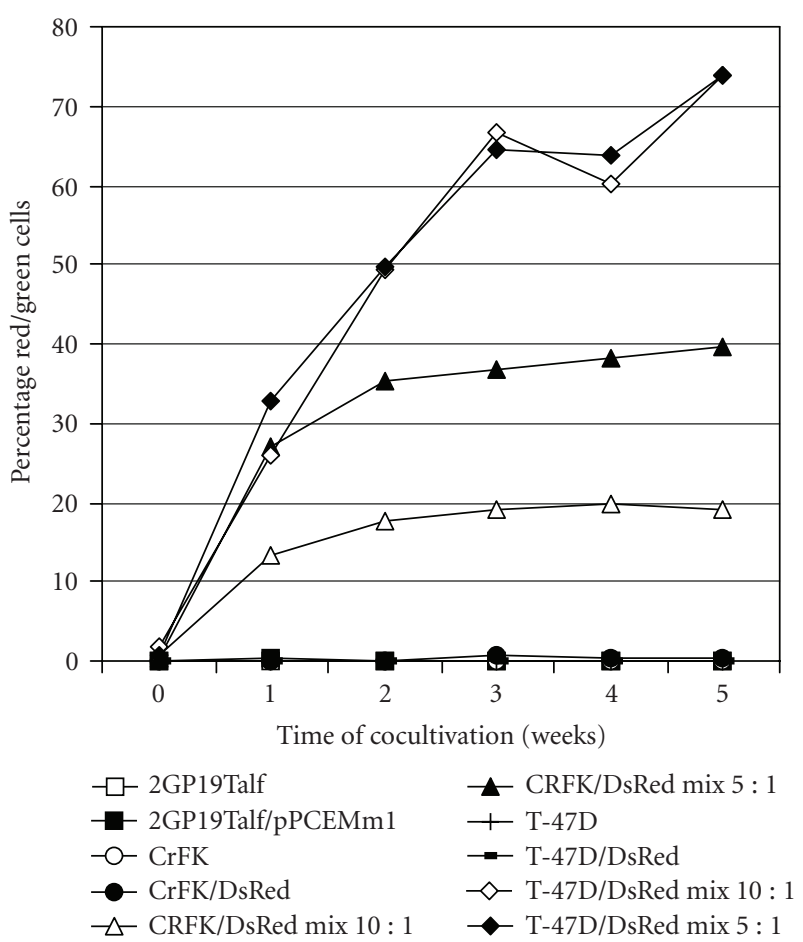

(a)

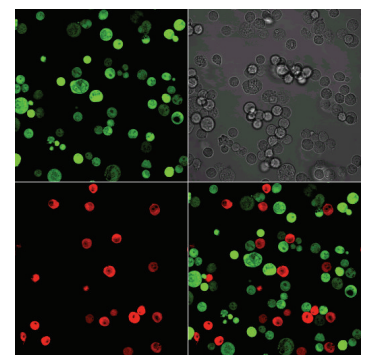

293/pPCEMm1 + CRFK/DsRed

(b)

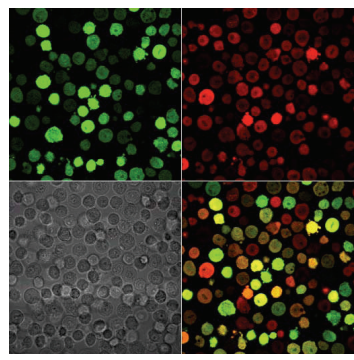

2GP19Talf/pPCEMm1 + CRFK/DsRed

(c)
Figure 2: In vitro infection of T-47D/DsRed and CRFK/DsRed cells with pPCEMm1. (a) FACS analysis of CRFK/DsRed and T47D/DsRed cells cocultivated with 2GP19Talf cells stably transfected with pPCEMm1 over a time period of five weeks. The numbers of red, green, and red/green fluorescent cells of a representative experiment are shown. As negative controls, 2GP19Talf, T-47D, and CRFK cells were used. As positive controls, pure 2GP19Talf/pPCEMm1, T-47D/DsRed, and CRFK/DsRed cells were used. (b) Confocal laser-scanning microscopy of CRFK/DsRed cells cocultivated with 293/pPCEMm1 cells over a time period of five weeks. Upper left (UL), green fluorescence; upper right (UR), transmission; lower left (LL), red fluorescence; lower right (LR), UL + LL. Magnification $400 \mathrm{x}$. (c) Confocal laser-scanning microscopy of CRFK/DsRed cells cocultivated with 2GP19Talf/pPCEMm1cells over a time period of five weeks. Upper left (UL), green fluorescence; upper right (UR), red fluorescence; lower left (LL), transmission; lower right (LR), UL + UR. Magnification $400 \mathrm{x}$.

expression of the eGFP reporter gene from an MLV promoter/CMV enhancer cassette while in infected cells after promoter conversion, expression of the eGFP gene is driven 
by the heterologous MMTV promoter.

For in vitro infection studies, 2GP19Talf virus-producing cells that had stably been transfected with pPCEMm1 were mixed in ratios of $1: 10$ or $1: 5$ with red fluorescent human T-47D breast cancer cells and as a control with red fluorescent feline CRFK kidney cells that are known to effectively being infected by retroviruses. Red fluorescence of target cells was achieved by stably transfecting the cells with the DsRed protein-encoding vector pCMV-dsRed-Express and enrichment of red fluorescent cells was performed by cell sorting. Mixed cells were cocultivated for five weeks and the numbers of green, red, and green/red cells were monitored by FACS (see Figure 2(a)). After five weeks, about $74 \%$ of red fluorescent T-47-D cells were also green fluorescent, indicating that they had been infected with pPCEMm1. The initial ratio between virus-producing cells and target cells did not play a role in this setting. The red fluorescent CRFK cells were infected to an extent of about 19\% (ratio 1:10 between virus-producing cells and target cells) and $40 \%$ (ratio $1: 5$ between virus-producing cells and target cells). In contrast, when green fluorescent nonvirus-producing 293 cells stably infected with pPCEMm1 where mixed with red fluorescent T-47D or CRFK cells, only a very low number $(<1 \%)$ of false-positive red/green cells were detected by FACS after six weeks of cocultivation (data not shown). The obtained results were also verified by confocal laser-scanning microscopy: no red/green double fluorescent cells could be detected as exemplified in Figure 2(b) for a mix of 293 cells stably infected with pPCEMm1 and CRFK/DsRed cells. In contrast, when the cell mixes of cocultivated red fluorescent T-47D or CRFK cells and virus-producing green fluorescent cells were analyzed, clearly a number of truly red/green double fluorescent cells could be detected as demonstrated for a mix of CRFK/DsRed and 2GP19Talf/pPCEMm1cells (see Figure 2(c)). Double fluorescence was not due to red autofluorescence of 2GP19Talf/pPCEMm1 or green autofluorescence of CRFK/pPCEMm 1 and T-47D/pPCEMm 1 cells since no red fluorescent cells could be detected in samples only consisting of green fluorescent 2GP19Talf/pPCEMm1 cells and no green fluorescence could be detected in samples of red fluorescent CRFK/DsRed and T-47D/DsRed cells (data not shown).

To investigate the in vivo infection capability of the MMTV promoter containing ProCon vector, mixed tumors consisting of DsRed-expressing T-47D or CRFK cells on the one hand and virus-producing 2GP19Talf/pPCEMm1 cells on the other hand were established in the mammary fat pads of nude mice. Again, the ratios between virus-producing cells and target cells at the time point of injection were $1: 10$ and $1: 5$. After 11 weeks of tumor growth, the tumors were explanted and analyzed by FACS (see Figure 3(a)). Of the red fluorescent T-47D cells of six mixed tumors, $22 \%$ to $55 \%$ were also green fluorescent, that is, had been infected with pPCEMm 1 . A range of $13 \%-33 \%$ of red fluorescent CRFK cells isolated from mixed tumors also showed green fluorescence, indicating infection by pPCEMm1. In addition, cells were analyzed by confocal laser-scanning microscopy (see Figures 3(b) and 3(c)). Clearly, cells fluorescing red as well as green were visible in the samples derived from mixed T-47D

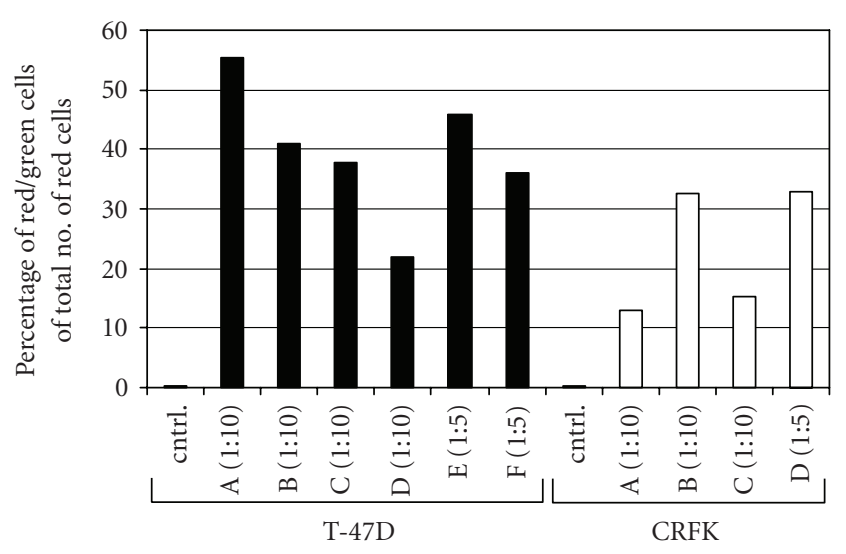

(a)

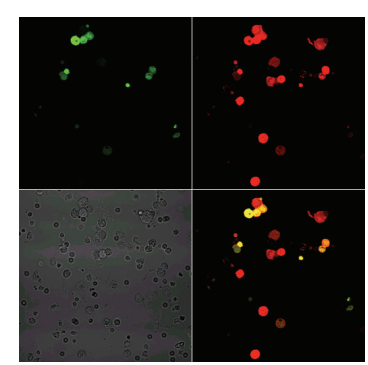

(b)

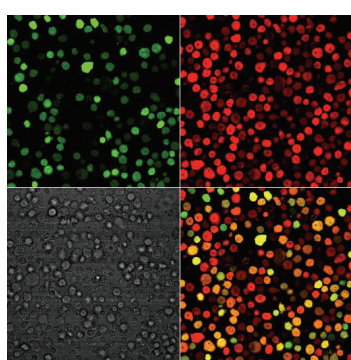

(c)
FIGURE 3: In vivo infection of T-47D/DsRed and CRFK/DsRed cells with pPCEMm1. (a) FACS analysis of cells from explanted mixed tumors consisting of 2GP19Talf/pPCEMm1 cells mixed with either T-47D/DsRed or CRFK/DsRed cells. The numbers of red, green, and red/green fluorescent cells were determined. Percentages of $\mathrm{red} /$ green doublefluorescent cells for individual tumors are shown. Individual tumors are designated with alphabetical characters below each bar. (b)-(c) Confocal laser-scanning microscopy of cells from explanted mixed tumors consisting of 2GP19Talf/pPCEMm1 and either T-47D/DsRed (b) or CRFK/DsRed cells (c). Upper left (UL), green fluorescence; upper right (UR), red fluorescence; lower left (LL), transmission; lower right (LR), UL + UR. Magnification $400 \mathrm{x}$.

(see Figure 3(b)) as well as from mixed CRFK tumors (see Figure $3(\mathrm{c})$ ). As expected, cells originating from control tumors solely consisting of 2GP19Talf/pPCEMm1 cells showed only green but no red fluorescence while cells stemming from control tumors exclusively consisting of T-47D/DsRed or CRFK/DsRed cells showed only red but no green fluorescence. Cells derived from control tumors only consisting of 2GP19Talf, T-47D, or CRFK cells exhibited no fluorescence at all (data not shown).

\subsection{The ProCon vector/virus packaging cell system is functional}

The eGFP reporter gene was replaced with the therapeutic gene coding for cytochrome P450 2B1 (CYP2B1). The resulting vector pPCCMm1 (see Figure 1) was used to transfect 2GP19Talf cells and vector harboring cells were selected 


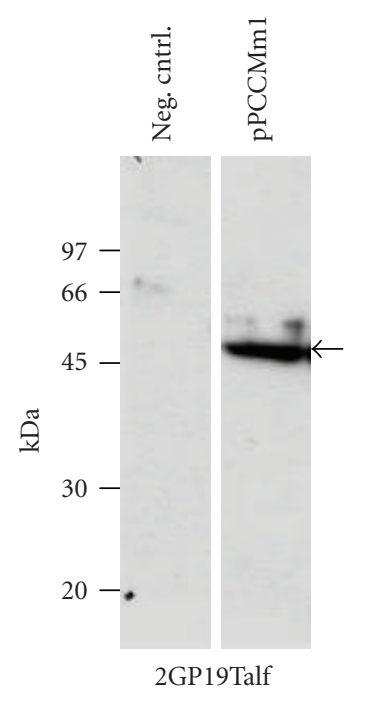

(a)

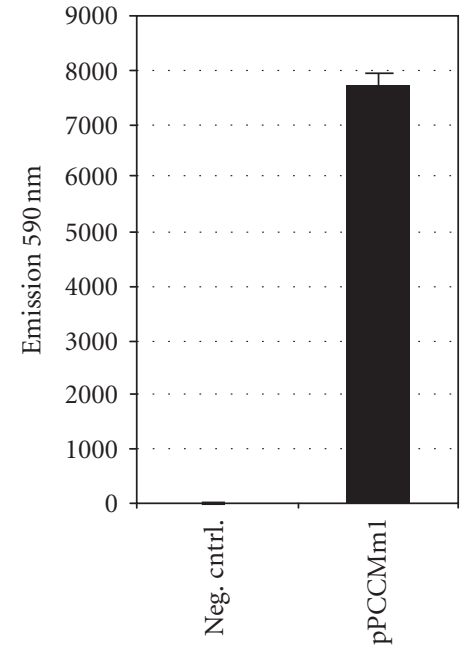

(b)

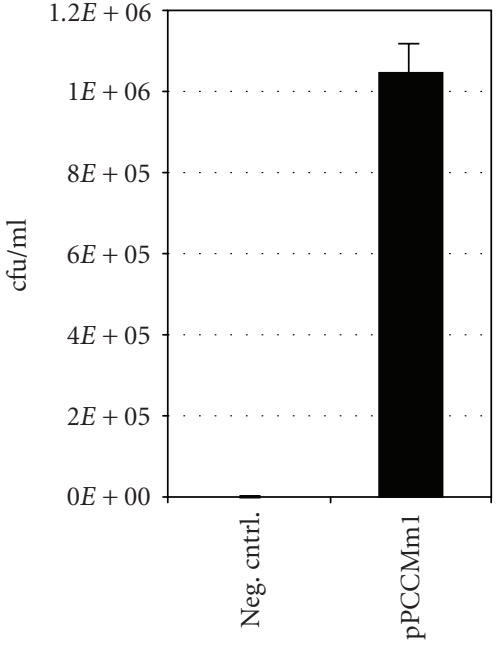

(c)

Figure 4: CYP2B1 expression, activity, and virus titers of 2GP19Talf amphotropic retroviral packaging cells stably transfected with pPCCMm1. (a) Expression of CYP2B1 in 2GP19Talf/pPCCMm1 cells. Cell lysates (equal amounts of protein) were analyzed by Western blotting for the presence of CYP2B1 using an anti-CYP2B1 antibody. The CYP2B1 band is indicated with an arrow. The molecular weights of the protein standard are given on the left side. Neg. cntrl.: 2GP19Talf cells not transfected with pPCCMm1. (b) Enzymatic activity of CYP2B1 in 2GP19Talf/pPCCMm1 cells. The enzymatic activity as determined by a resorufin assay is shown as the relative emission intensity of the created product resorufin at $590 \mathrm{~nm}$ in comparison to nontransfected cells (neg. cntrl.). (c) Virus titers of 2GP19Talf/pPCCMm1 cells as determined by counting the numbers of colonies of infected G418-resistant CRFK cells (colony-forming units per milliliter; CFU/mL). Two independent infection experiments were performed. Plating of dilutions of cells after infection was performed in triplicates, each. Neg. cntrl.: 2GP19Talf cells not transfected with pPCCMm1.

with geneticin in order to obtain a population of stably transfected cells. Western blot analysis was used to prove that CYP2B1 was produced in 2GP19Talf/pPCCMm1 cells (see Figure 4(a)). To not only test for the presence of the protein but also for its activity, resorufin formation as a consequence of CYP2B1 activity was monitored using a 96-well format assay. Generation of resorufin in 2GP19Talf/pPCCMm 1 cells in relation to 2GP19Talf negative control cells after incubation with pentoxyresorufin is shown in Figure 4(b). The presence of resorufin indicated that CYP2B1 was active.

For infection of target tumor cells, sufficiently high numbers of virus particles have to be generated by the virusproducing cells. Virus titers were determined by infection of CRFK cells with virus particles from the supernatant of 2GP19Talf/pPCCMm1 cells and followed by counting the numbers of G418 resistant colonies. Titers were about $1.3 \times$ $10^{6}$ colony-forming units $(\mathrm{CFU}) / \mathrm{mL}$ of cell supernatant (see Figure $4(\mathrm{c})$ ) which is comparable to titers obtained with the respective eGFP gene-containing vector pPCEMm1 [27].

\subsection{The MMTV promoter ProCon system generates a pronounced cell killing effect in vitro}

As a model breast cancer cell line to study the efficacy of our vector system, the T-47D cell line was chosen. T-47D cells were infected with pPCCMm1 and stable populations were created by selection of infected cells with G418. For induction of gene expression from the MMTV promoter cells were stimulated with dexamethasone for 48 hours and analyzed by Western blotting for the presence of CYP2B1. While an antiCYP2B1 antibody did not detect any CYP2B1 in noninfected T-47D cells (negative control), CYP2B1 was clearly present in T-47D cells infected with pPCCMm1 (see Figure 5(a)). Resorufin assays revealed that the protein was also active in that cell line (Figure 5(b)). Enzyme activities were 2.5-fold higher in cells that had been treated with dexamethasone compared to those in nontreated cells (data not shown).

To evaluate the sensitizing effect of CYP2B1 on T-47D cells upon treatment with the anticancer prodrug ifosfamide, T-47D cells infected with pPCCMm 1 and noninfected T-47D control cells were treated with increasing amounts of ifosfamide (ranging from 0 to $3 \mathrm{mM}$ ) for five consecutive days after which cell viabilities were determined using an XTT assay. While noninfected T-47D cells remained largely unaffected up to an ifosfamide concentration of $0.3 \mathrm{mM}$, T47D/pPCCMm1 cells expressing the CYP2B1 gene showed a marked decrease of $40 \%$ already at a concentration of $0.1 \mathrm{mM}$. This viability decreased further at higher concentrations (see Figure 6(a)). $0.3 \mathrm{mM}$ ifosfamide was determined as the most effective concentration at which noninfected T-47 cells were still largely unaffected but CYP2B1-producing T47D/pPCCMm1 cells showed a dramatic decrease in viability 


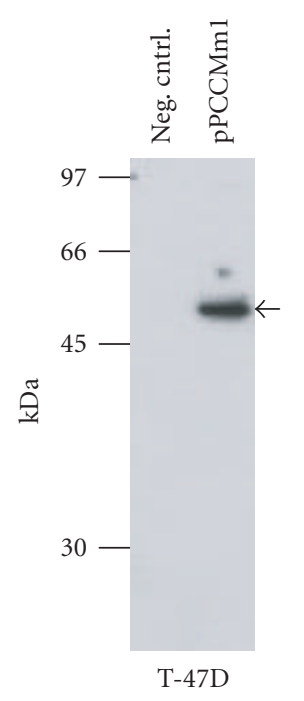

(a)

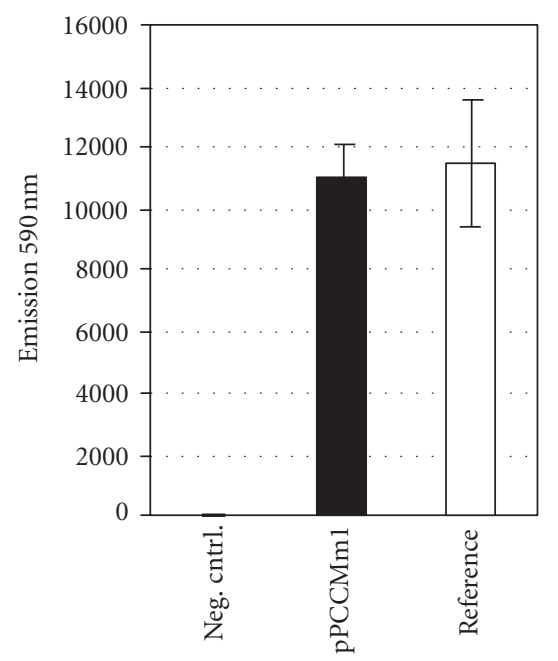

(b)

Figure 5: CYP2B1 expression and activity in T-47D cells stably infected with pPCCMm1. (a) Expression of CYP2B1 in a population of T-47D/pPCCMm1 cells. Cell lysates (equal amounts of protein) were analyzed by Western blotting for the presence of CYP2B1 using an anti-CYP2B1 antibody. The CYP2B1 band is indicated with an arrow. The molecular weights of the protein standard are given on the left side. Neg. cntrl.: T-47D cells not infected with pPCCMm1. (b) Enzymatic activity of CYP2B1 in a population of T-47D/pPCCMm1 cells. The enzymatic activity as determined by a resorufin assay is shown as the relative emission intensity of the created product resorufin at $590 \mathrm{~nm}$ in comparison to noninfected T-47D cells (neg. cntrl.) and a reference clone of virus-packing cells (22P1G).

by $88 \%$. IC $\mathrm{C}_{50}$ values were determined as $1.6 \mathrm{mM}$ and $0.1 \mathrm{mM}$ for T-47D and T-47D/pPCCMm1, respectively. This represents a decrease of the $\mathrm{IC}_{50}$ value by sixteen folds, underlining the potent cumulative cytotoxic effect of CYP2B1 and ifosfamide. Treatment of the cells with cyclophosphamide instead of ifosfamide led to similar results (see Figure 6(b)).

\section{DISCUSSION}

The ProCon vectors we have generated in this study are a further development of an earlier, a $\beta$-galactosidase reporter gene-containing vector that was used for the generation of virus-producing cells for encapsulation experiments [19]. The new vectors contain modifications that have recently been shown by our laboratory to improve their overall performance (i.e., a CMV enhancer to increase viral RNA production in virus-producing cells, a strong polyadenylation signal in the modified $3^{\prime}$ LTR to prevent read-through of viral RNA and to stabilize the mRNA, and an elongated attachment site to increase the integration efficiency of the provirus [26]). When transferred into virus packaging cells, our newly generated vectors allow the production of enzymatically active CYP2B1 that converts ifosfamide or cyclophosphamide into their tumoricidal metabolites. As demonstrated by Western blotting and with resorufin assays, a high concentration of CYP2B1 in virus-producing cells is ascertained by the strong MLV promoter that drives the expression of the therapeutic gene. Similarly, when incorporating the eGFP reporter gene into the vectors, high MFIs in
FACS analyses can be achieved in virus-producing cells. In contrast to what was seen with similar vectors containing the WAP promoter [27], when incorporated into our new vectors, the woodchuck hepatitis virus posttranscriptional regulatory element (WPRE) enhanced the expression rate of both the eGFP and the CYP2B1 genes in virus packaging cells as well as in infected cells (data not shown). Therefore, vectors pPCCMm1 and pPCEMm1 harboring the WPRE were chosen for further experiments over versions of those vectors lacking the WPRE (data not shown).

pPCCMm1 and the reporter gene-harboring vector pPCEMm1 allow the generation of high virus titers in viruspackaging cells and productive infection of target cells in vitro. In cocultivation experiments employing virus packaging cells and target cells, the infection rates of T-47D and CRFK target cells reached $74 \%$ and $40 \%$, respectively. The lower infection rate of the CRFK cells was due to the higher growth rate of the CRFK cells compared to that of the 2GP19Talf/pPCEMm1 virus-packaging cells, quickly leading to the displacement of the latter ones. As a consequence, virus particle numbers in the cell supernatants decreased over time. The infection rates were also reflected by similar results obtained with confocal laser-scanning microscopy, demonstrating true infection of target cells. The small numbers of double fluorescent cells $(<1 \%)$ obtained after six weeks of cocultivation of red fluorescent target cells and green fluorescent nonvirus-producing cells represented false-positive cells since no such double fluorescent cells were observed in laser-scanning microscopy. Most likely, the small numbers 


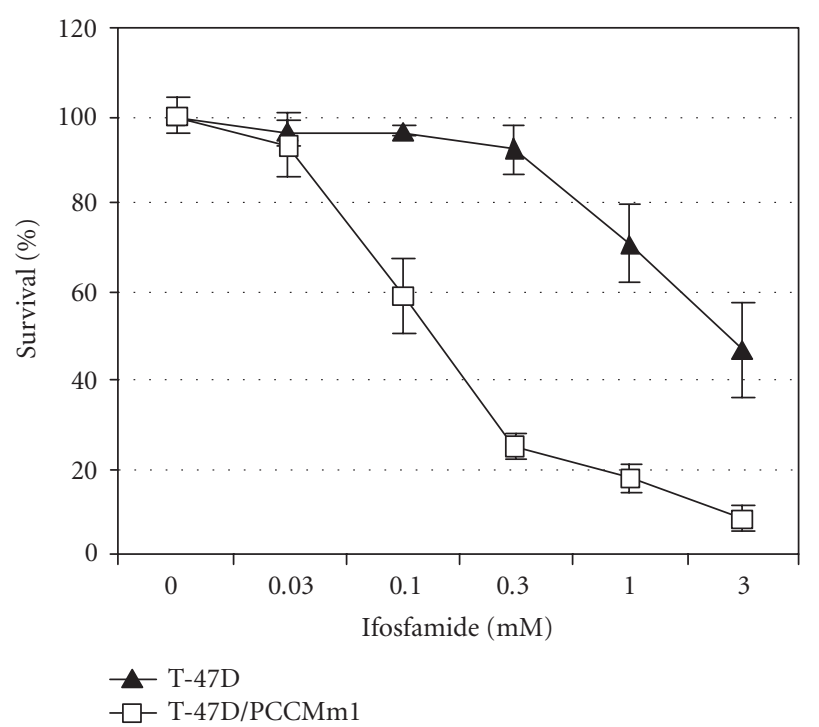

(a)

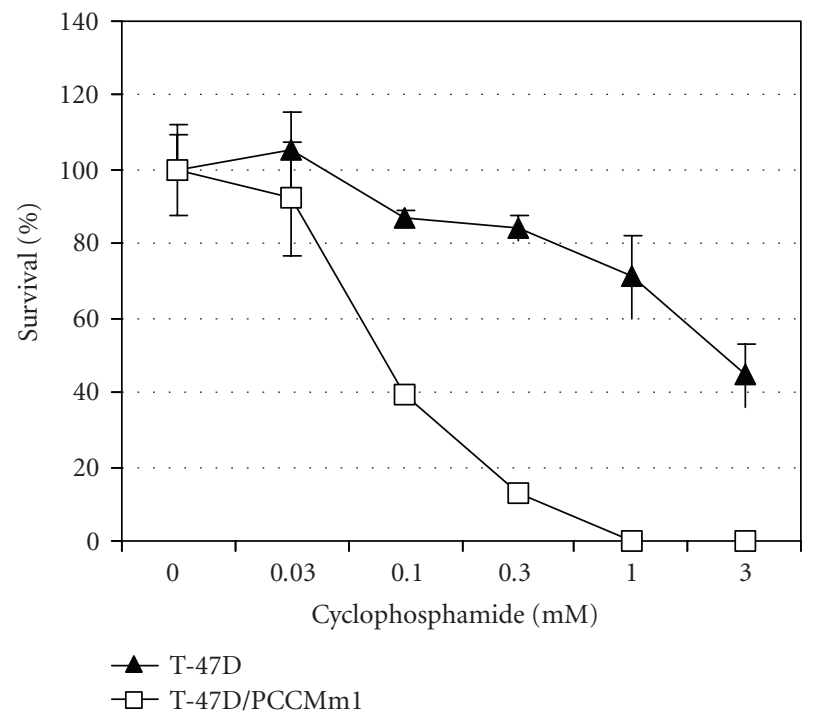

(b)

FIGURE 6: Enhanced cytotoxicities of ifosfamide and cyclophosphamide in T-47D/pPCCMm1 cells expressing CYP2B1. Cells were cultivated at increasing amounts of ifosfamide for five days as described in materials and methods. Cell viabilities were determined using an XTT assay and survival rates of CYP2B1 expressing cells in relation to noninfected control cells were calculated. (a) Incubation of cells with ifosfamide. (b) Incubation of cells with cyclophosphamide.

of false-positive red/green cells observed in FACS experiments were rather due to small numbers of red and green cells sticking together, thus, giving the impression of doublefluorescent cells. This is also underlined by the fact that small numbers of red/green fluorescent cells were even detected when red fluorescent cells were mixed with green fluorescent cells immediately prior to FACS (data not shown).

Since in vivo infection may be less efficient compared to invitro infection, we tested our vectors in a mixed tumor model in nude mice in vivo. Analysis of explanted tumors revealed that $22 \%$ to $55 \%$ of T-47D target cells and $13 \%$ to $33 \%$ of CRFK target cells had been infected with vector pPCEMm1. The somewhat lower infection rate for CRFK cells was probably due to their excellent growth rate in vivo compared to the poor growth rate of the 293-based virusproducing cells and a subsequent shift in the ratio between virus-producing cells and target cells in favor of the target cells. This probably led to a substantial decrease of virus titer within the tumor over time. However, this will not be an issue if vector-producing cells will be encapsulated. In addition, an infection rate of $100 \%$ is not necessarily required during a therapy employing encapsulated viral vector-producing cells because of the bystander effect of cyclophosphamide or ifosfamide on neighboring noninfected cells in GDEPT $[6,33]$.

Incorporation of the MMTV promoter into the retroviral vectors allowed high transgene expression of the eGFP reporter gene or the therapeutic CYP2B1 gene in infected cells. This was not only seen for T-47D and CRFK cells but also for a number of other human or nonhuman cell lines (mainly of mammary origin; data not shown). In most of the cell lines, transgene expression could be further boosted by stimulation with the glucocorticoid dexamethasone. Trans- gene expression was also increased in most of the cell lines when cells were treated with progesterone, although the effect was less pronounced (data not shown). Dexamethasone is frequently used in cancer chemotherapy, either as a tumoricidal therapeutic $[34,35]$ or to prevent or diminish nausea and other side effects in patients (including breast cancer patients) undergoing chemotherapy $[36,37]$. Thus, in a GDEPT setting, dexamethasone would decrease the symptoms of the side effects of the chemotherapy and at the same time increase the expression of the therapeutic gene from the MMTV promoter.

In this study, we also showed that the level of enzymatically active CYP2B1 generated in infected cells with the newly developed vectors is pronounced enough to create a highly increased cell killing effect on T-47D/pPCCMm1 cells upon ifosfamide or cyclophosphamide treatment in vitro. The enhanced cytotoxic effect due to infection with pPC$\mathrm{CMm} 1$ became manifest in a sixteen fold-lowered $\mathrm{IC}_{50}$ value for ifosfamide in T-47D/pPCCMm1 compared to noninfected T-47D cells. This value was similar for cyclophosphamide. Effective concentrations of 0.1-0.5 mM ifosfamide have been measured in the plasma of patients after administration of typical dosages of ifosfamide during conventional chemotherapy [38]. These pharmacologically active concentrations are identical to those we determined, which resulted in efficient killing of CYP2B1-expressing T-47D cells while leaving T-47D cells not expressing CYP2B1 largely unaffected.

Taken together, the newly generated vectors constitute the basis for the development of a novel breast cancer GDEPT system: the system is anticipated to employ the encapsulation of cells that (i) generate the therapeutic enzyme 
CYP2B1 and, (ii) in addition, produce viral vector particles that transfer the therapeutic CYP2B1 gene directly into tumor cells.

\section{ACKNOWLEDGMENT}

The authors thank Dr. Harry Holzmüller, Austrianova Biotechnology $\mathrm{GmbH}$, for providing plasmid pPCCmCMV .WPRE.

\section{REFERENCES}

[1] S. R. McKeown, C. Ward, and T. Robson, "Gene-directed enzyme prodrug therapy: a current assessment," Current Opinion in Molecular Therapeutics, vol. 6, no. 4, pp. 421-435, 2004.

[2] N. E. Sladek, "Metabolism and pharmacokinetic behavior of cyclophosphamide and related oxazaphosphorines," in Anticancer Drugs: Reactive Metabolism and Interactions, G. Powers, Ed., pp. 79-156, Pergamon Press, Oxford, UK, 1994.

[3] L. Clarke and D. J. Waxman, "Oxidative metabolism of cyclophosphamide: identification of the hepatic monooxygenase catalysts of drug activation," Cancer Research, vol. 49, no. 9, pp. 2344-2350, 1989.

[4] T. K. Chang, G. F. Weber, C. L. Crespi, and D. J. Waxman, "Differential activation of cyclophosphamide and ifosphamide by cytochromes P-450 2B and 3A in human liver microsomes," Cancer Research, vol. 53, no. 23, pp. 5629-5637, 1993.

[5] L. Chen and D. J. Waxman, "Intratumoral activation and enhanced chemotherapeutic effect of oxazaphosphorines following cytochrome P-450 gene transfer: development of a combined chemotherapy/cancer gene therapy strategy," Cancer Research, vol. 55, no. 3, pp. 581-589, 1995.

[6] M. X. Wei, T. Tamiya, R. J. Rhee, X. O. Breakefield, and E. A. Chiocca, "Diffusible cytotoxic metabolites contribute to the in vitro bystander effect associated with the cyclophosphamide/cytochrome P450 2B1 cancer gene therapy paradigm," Clinical Cancer Research, vol. 1, no. 10, pp. 11711177, 1995.

[7] L. Hennighausen, "Mouse models for breast cancer," Breast Cancer Research, vol. 2, no. 1, pp. 2-7, 2000.

[8] T. A. Stewart, P. K. Pattengale, and P. Leder, "Spontaneous mammary adenocarcinomas in transgenic mice that carry and express MTV/myc fusion genes," Cell, vol. 38, no. 3, pp. 627637, 1984.

[9] S. R. Ross and D. Solter, "Glucocorticoid regulation of mouse mammary tumor virus sequences in transgenic mice," Proceedings of the National Academy of Sciences of the United States of America, vol. 82, no. 17, pp. 5880-5884, 1985.

[10] K.-U. Wagner, R. J. Wall, L. St-Onge, et al., "Cre-mediated gene deletion in the mammary gland," Nucleic Acids Research, vol. 25, no. 21, pp. 4323-4330, 1997.

[11] F. Ahmed, J. Wyckoff, E. Y. Lin, et al., "GFP expression in the mammary gland for imaging of mammary tumor cells in transgenic mice," Cancer Research, vol. 62, no. 24, pp. 71667169, 2002.

[12] E. R. Andrechek, M. A. Laing, A. A. Girgis-Gabardo, P. M. Siegel, R. D. Cardiff, and W. J. Muller, "Gene expression profiling of neu-induced mammary tumors from transgenic mice reveals genetic and morphological similarities to ErbB2expressing human breast cancers," Cancer Research, vol. 63, no. 16, pp. 4920-4926, 2003.
[13] B. Muñoz and F. F. Bolander Jr., "Prolactin regulation of mouse mammary tumor virus (MMTV) expression in normal mouse mammary epithelium," Molecular and Cellular Endocrinology, vol. 62, no. 1, pp. 23-29, 1989.

[14] J. Svec and J. Links, "Mouse mammary tumor virus production stimulated by hormones and polyamines in cells grown in semi-synthetic in vitro conditions," International Journal of Cancer, vol. 19, no. 2, pp. 249-257, 1977.

[15] J. Ham, A. Thomson, M. Needham, P. Webb, and M. Parker, "Characterization of response elements for androgens, glucocorticoids and progestins in mouse mammary tumour virus," Nucleic Acids Research, vol. 16, no. 12, pp. 5263-5276, 1988.

[16] E. Weber, W. F. Anderson, and N. Kasahara, "Recent advances in retrovirus vector-mediated gene therapy: teaching an old vector new tricks," Current Opinion in Molecular Therapeutics, vol. 3, no. 5, pp. 439-453, 2001.

[17] J. E. McCormack, W. Edwards, J. Sensintaffer, et al., "Factors affecting long-term expression of a secreted transgene product after intravenous administration of a retroviral vector," Molecular Therapy, vol. 3, no. 4, pp. 516-525, 2001.

[18] B. Salmons and W. H. Gunzburg, "Targeting of retroviral vectors for gene therapy," Human Gene Therapy, vol. 4, no. 2, pp. 129-141, 1993.

[19] R. M. Saller, S. Indraccolo, V. Coppola, et al., "Encapsulated cells producing retroviral vectors for in vivo gene transfer," Journal of Gene Medicine, vol. 4, no. 2, pp. 150-160, 2002.

[20] O. Martinet, N. Schreyer, E. D. Reis, and J.-M. Joseph, "Encapsulation of packaging cell line results in succesful retroviralmediated transfer of a suicide gene in vivo in an experimental model of glioblastoma," European Journal of Surgical Oncology, vol. 29, no. 4, pp. 351-357, 2003.

[21] A. Krupetsky, Z. Parveen, E. Marusich, A. Goodrich, and R. Dornburg, "Retroviral packaging cells encapsulated in theracyte immunoisolation devices enable long-term in vivo gene delivery," Frontiers in Bioscience, vol. 8, pp. a94-a101, 2003.

[22] M. Löhr, P. Müller, P. Karle, et al., "Targeted chemotherapy by intratumour injection of encapsulated cells engineered to produce CYP2B1, an ifosfamide activating cytochrome P450," Gene Therapy, vol. 5, no. 8, pp. 1070-1078, 1998.

[23] T. Kammertoens, W. Gelbmann, P. Karle, et al., "Combined chemotherapy of murine mammary tumors by local activation of the prodrugs ifosfamide and 5-fluorocytosine," Cancer Gene Therapy, vol. 7, no. 4, pp. 629-636, 2000.

[24] B. Salmons, M. Löhr, and W. H. Günzburg, "Treatment of inoperable pancreatic carcinoma using a cell-based local chemotherapy: results of a phase I/II clinical trial," Journal of Gastroenterology, vol. 38, supplement 15, pp. 78-84, 2003.

[25] M. Löhr, A. Hoffmeyer, J.-C. Kröger, et al., "Microencapsulated cell-mediated treatment of inoperable pancreatic carcinoma," The Lancet, vol. 357, no. 9268, pp. 1591-1592, 2001.

[26] J. Hlavaty, A. Stracke, D. Klein, B. Salmons, W. H. Günzburg, and M. Renner, "Multiple modifications allow high-titer production of retroviral vectors carrying heterologous regulatory element," Journal of Virology, vol. 78, no. 3, pp. 1384-1392, 2004.

[27] R. Klein, B. Ruttkowski, E. Knapp, B. Salmons, W. H. Günzburg, and C. Hohenadl, "WPRE-mediated enhancement of gene expression is promoter and cell line specific," Gene, vol. 372, pp. 153-161, 2006.

[28] K. Pambalk, C. Hohenadl, B. Salmons, W. H. Günzburg, and M. Renner, "Specific packaging of spliced retroviral vector transcripts lacking the $\psi$-region," Biochemical and Biophysical Research Communications, vol. 293, no. 1, pp. 239-246, 2002. 
[29] F. L. Graham, J. Smiley, W. C. Russell, and R. Nairn, "Characteristics of a human cell line transformed by DNA from human adenovirus type 5," Journal of General Virology, vol. 36, no. 1, pp. 59-72, 1977.

[30] R. A. Crandell, C. G. Fabricant, and W. A. Nelson-Rees, "Development, characterization, and viral susceptibility of a feline (Felis catus) renal cell line (CRFK)," In Vitro, vol. 9, no. 3, pp. 176-185, 1973.

[31] I. Keydar, L. Chen, S. Karby, et al., "Establishment and characterization of a cell line of human breast carcinoma origin," European Journal of Cancer \& Clinical Oncology, vol. 15, no. 5, pp. 659-670, 1979.

[32] W. H. Günzburg, P. Karle, R. Renz, B. Salmons, and M. Renner, "Characterization of a human cell clone expressing cytochrome P450 for safe use in human somatic cell therapy," Annals of the New York Academy of Sciences, vol. 880, pp. 326336, 1999.

[33] L. Chen, D. J. Waxman, D. Chen, and D. W. Kufe, "Sensitization of human breast cancer cells to cyclophosphamide and ifosfamide by transfer of a liver cytochrome P450 gene," Cancer Research, vol. 56, no. 6, pp. 1331-1340, 1996.

[34] P. S. Gaynon and A. L. Carrel, "Glucocorticosteroid therapy in childhood acute lymphoblastic leukemia," Advances in Experimental Medicine and Biology, vol. 457, pp. 593-605, 1999.

[35] S. Greenstein, K. Ghias, N. L. Krett, and S. T. Rosen, "Mechanisms of glucocorticoid-mediated apoptosis in hematological malignancies," Clinical Cancer Research, vol. 8, no. 6, pp. 16811694, 2002.

[36] R. Sharma, P. Tobin, and S. J. Clarke, "Management of chemotherapy-induced nausea, vomiting, oral mucositis, and diarrhoea," The Lancet Oncology, vol. 6, no. 2, pp. 93-102, 2005.

[37] K. Jordan, C. Kasper, and H.-J. Schmoll, "Chemotherapyinduced nausea and vomiting: current and new standards in the antiemetic prophylaxis and treatment," European Journal of Cancer, vol. 41, no. 2, pp. 199-205, 2005.

[38] J. E. Wright, O. Tretyakov, L. J. Ayash, A. Elias, A. Rosowsky, and E. Frei, "Analysis of 4-hydroxycyclophosphamide in human blood," Analytical Biochemistry, vol. 224, no. 1, pp. 154158, 1995. 


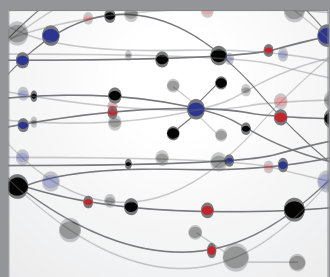

The Scientific World Journal
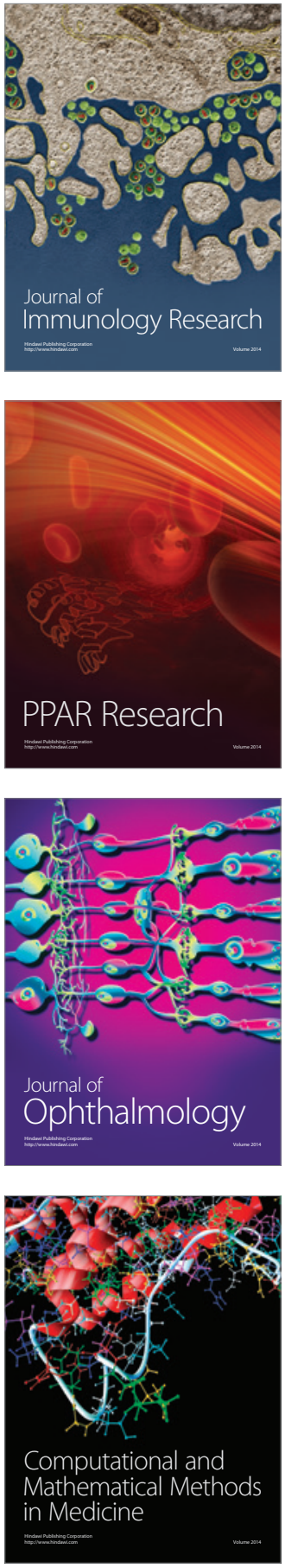

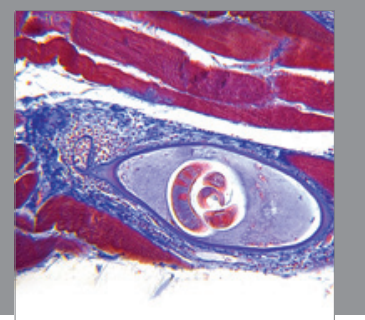

Gastroenterology

Research and Practice
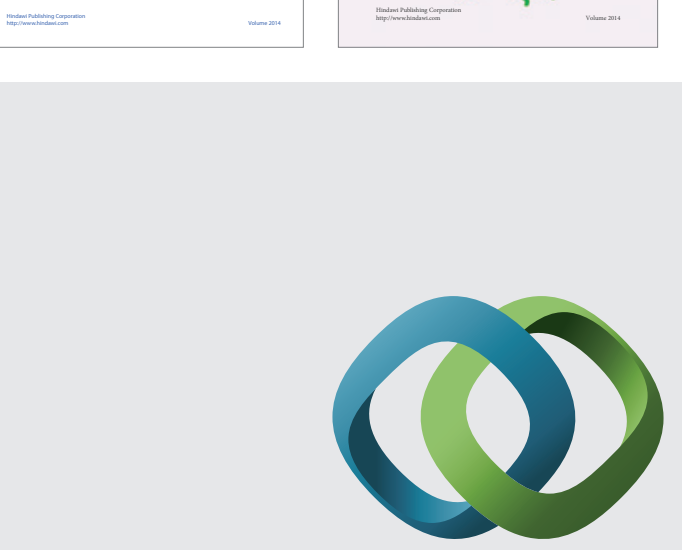

\section{Hindawi}

Submit your manuscripts at

http://www.hindawi.com
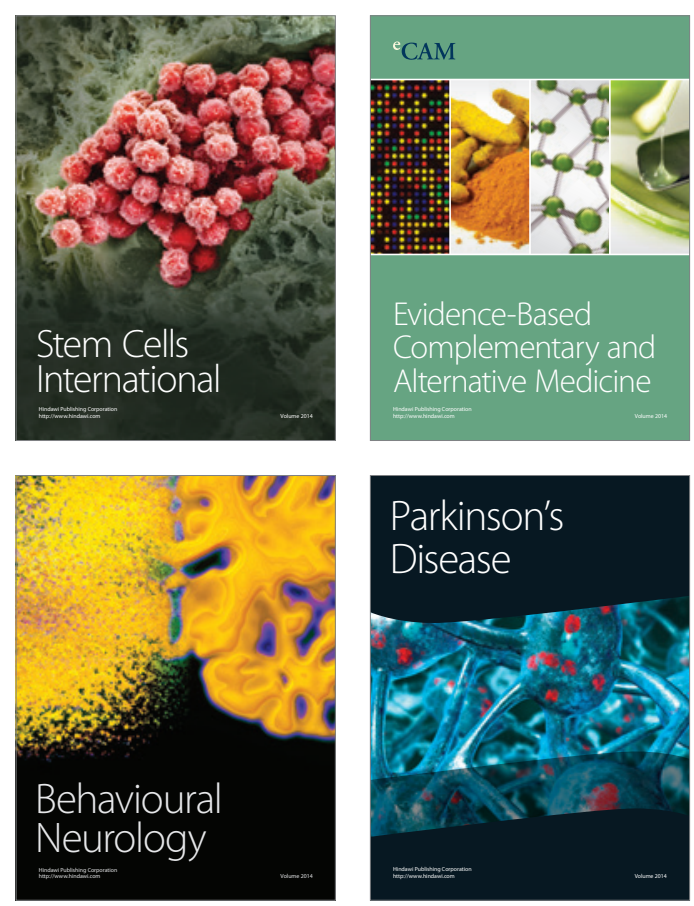

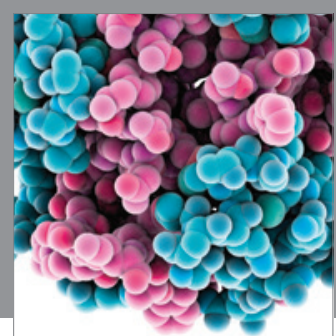

Journal of
Diabetes Research

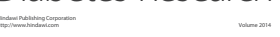

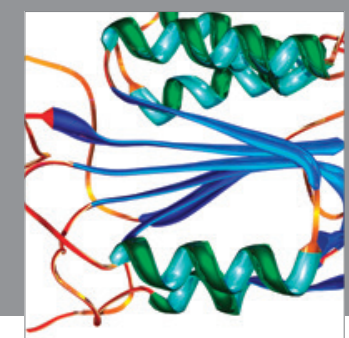

Disease Markers
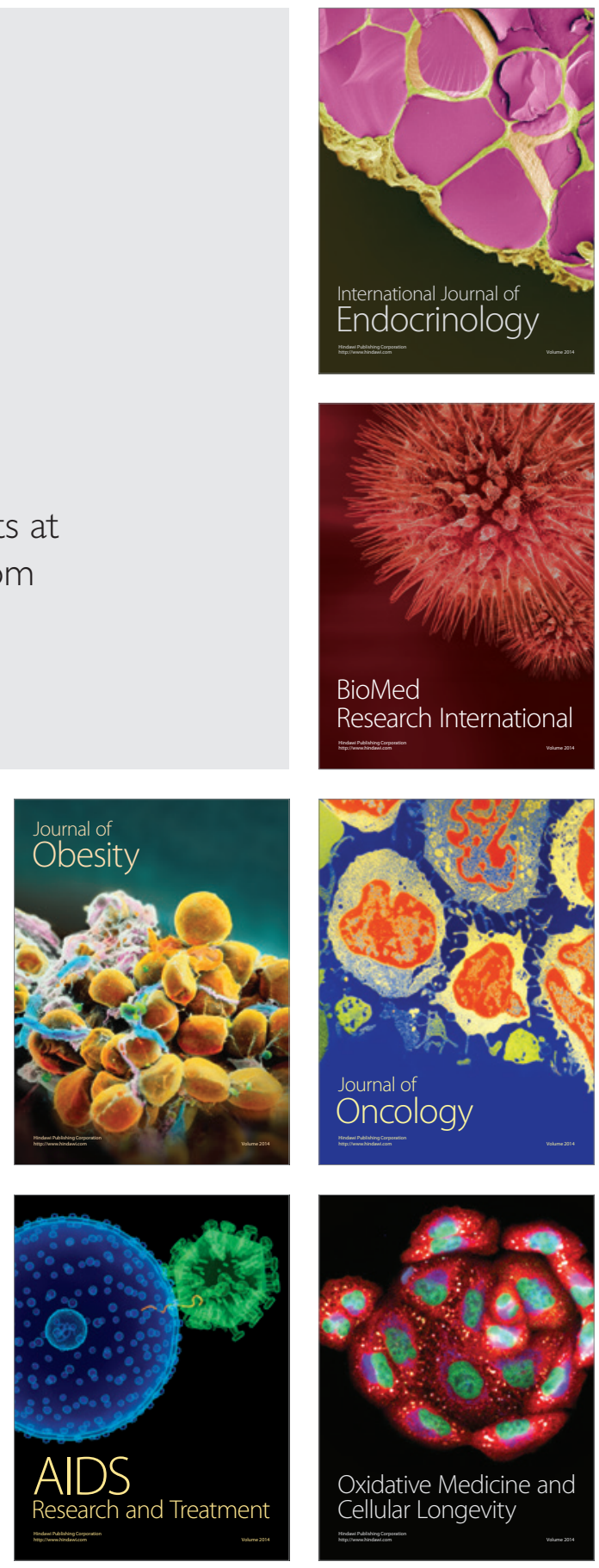